\title{
Synthesis and magnetic properties of stable nitroxyl tri-radicals
}

\author{
Mong-Jong Tien, ${ }^{a} \mathrm{Yu}$ Wang, ${ }^{a}$ Tong-Ing $\mathrm{Ho},{ }^{a}, *$ and Long $\mathrm{Y}$. Chiang ${ }^{\mathrm{b}}$ \\ a Department of Chemistry and ${ }^{b}$ Center for Condensed Matter Sciences, \\ National Taiwan University, Taipei, Taiwan
}

\begin{abstract}
Several stable nitroxyl tri-radicals, such as tris- $\alpha, \alpha^{\prime}, \alpha^{\prime \prime}-(t$-butylnitroxyl alkyl)mesitylene (1) and $N, N^{\prime}, N^{\prime \prime}$-mesitylenylidenetris (4-amino-2,2,6,6-tetramethylpiperidinyloxy) (2), were synthesized by either an addition reaction of alkyl Grignard reagents with corresponding trinitrone molecules or the condensation reaction of 4-amino-2,2,6,6-tetramethylpiperidinyloxy with 1,3,5-benzenetricarboxaldehyde (trimesic aldehyde). The $\chi_{\mathrm{m}} T\left(\chi_{\mathrm{m}}=\right.$ molar magnetic susceptibility) value was found to increase

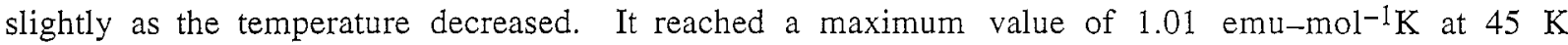
followed by a sharp decline of the $\chi_{\mathrm{m}} T$ value, indicating the existence of intermolecular antiferromagnetic interactions $(\theta=-1.91 \mathrm{~K})$ in this molecular system. The effective magnetic moment was found to be $2.85 \mu_{\mathrm{B}}$.
\end{abstract}

Keywords: tris- $\alpha, \alpha^{\prime}, \alpha^{\prime \prime}$-( $t$-butylnitroxyl alkyl)mesitylene; $N, N^{\prime}, N^{\prime \prime}$-mesitylenylidenetris (4-amino-2,2,6,6tetramethylpiperidinyloxy); nitroxide radicals.

\section{Introduction}

One of recent challenges in the field of molecular based ferromganetic materials is focused on the development of new molecular radicals [1]. For example, stable phenoxy1 radicals, including galvinoxyl radicals, have been found to exhibit ferromagnetic intermolecular spin interactions at temperatures below $7 \mathrm{~K}$ [2] Stability of phenoxyl radicals can be enhanced by introducing sterically hindered $t$-butyl group at adjacent positions of the radical center or by introducing oxygen-interactive cyano functions in conjugation with the phenoxy radical [3]. In our laboratory, we have prepared new radical materials and carried out electron spin resonance spectroscopic studies of some stable nitroxyl radicals [4], since stable nitroxyl radicals represent one of the most promising ferromagnetic materials [5]. They can be synthesized by known synthetic routes. It is known that relatively high symmetry and ground-state molecular orbital degeneracy in organic molecules are necessary for the stabilization of ferromagnetic couplings in the McConnell mechansm [5]. Stable radicals with $D_{2 \mathrm{~d}}, C_{3}$ or a higher symmetry are, therefore, among the most desirable target molecules to be synthesized. In this report, we present a synthetic scheme and ESR spectra of three new nitroxyl triradicals, $\mathbf{1}(\mathrm{a}-\mathrm{b})$ and 2 , using trimesic aldehyde as a starting reagent. Recently, trimesic aldehyde was utilized in the synthesis of a nitronyl nitroxide triradical (TNN) [6] which exhibited antiferromagnetic interactions between radicals in the bulk solid materials.

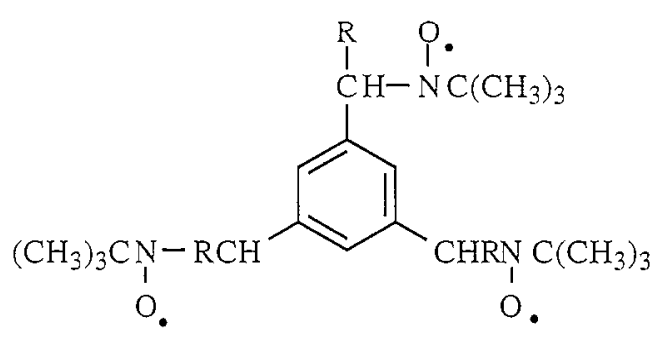

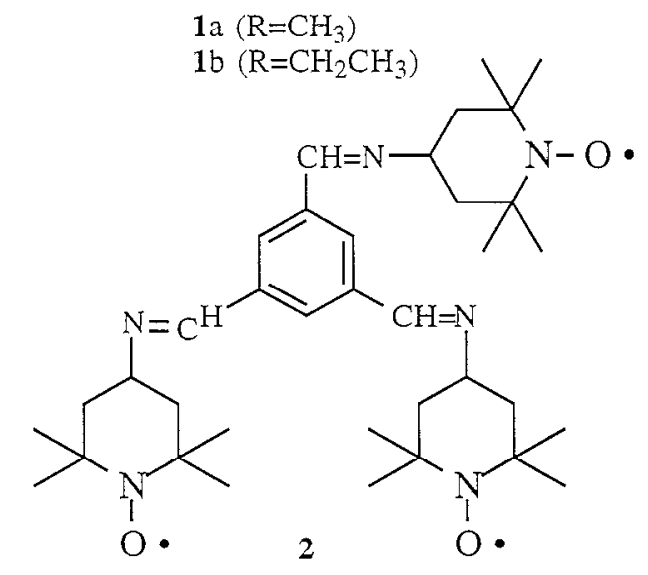<smiles>C[N+]([O-])=Cc1cc(C=[N+](C)C(C)(C)C)cc(C=[N+]([O-])C(C)(C)C)c1</smiles> 


\section{Results and Discussion}

Radical 1a and $\mathbf{1 b}$ were prepared by the reaction of trinitrone 3 with the corresponding methyl lithium or methylmagnesium bromide and ethylmagnesium bromide, respectively, as a reagent in the presence of an oxygen atmosphere (equation 1) [6]. Both radicals $\mathbf{1 a}$ and $\mathbf{1 b}$ showed triplets of doublet peaks in e.s.r. spectra at room temperature, as shown in Fig. 1.

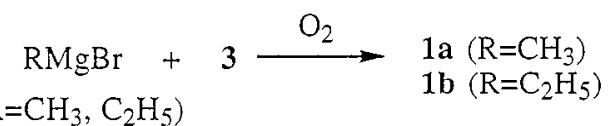

The hyperfine splitting constants of these radicals in interacting with nitrogen atom $\left(a_{\mathrm{N}}\right)$ and $\beta$-proton $\left(a_{\mathrm{H}}\right)$ were obtained by the analysis of their room temperature e.s.r. spectra; $a_{\mathrm{N}}=14.7 \mathrm{G}$ and $a_{\mathrm{II}}=3.6 \mathrm{G}$ for $1 \mathrm{a} ; a_{\mathrm{N}}=14.6 \mathrm{G}$ and $a_{\mathrm{II}}=3.6$ $\mathrm{G}$ for $1 \mathrm{~b}$. Apparently, there are no intramolecular spin-spin interactions among radical centers in $\mathbf{1}$ a and 1b. Unfortunately, we experienced difficulties in obtaining any sizable single crystal for X-ray analyses and magnetic susceptibility measurements. Radical 2 was prepared by the condensation reaction of 4-amino-2,2,6,6tetramethyl-piperidin-1-oxyl (4- $\mathrm{NH}_{2}$-TEMPO) with trimesic aldehyde [7] (equation 2). The e.s.r. spectrum of 2 was measured in benzene at room temperature, showing only three peak lines which correspond to hyperfine splitting constants arised from interactions of radical centers with a nitrogen atom with $a_{\mathrm{N}}=15.4 \mathrm{G}$ and $g=2.0062$.<smiles>CC1(C)CC(N)CC(C)(C)N1O</smiles><smiles>CCCC=O</smiles>

Temperature dependent magnetic susceptibilities of a microcrystalline sample 2 was measured at 2-300 $\mathrm{K}$ under a magnetic field of one tesla. The plot of $\chi_{\mathrm{m}} T$ ( $\chi_{\mathrm{m}}=$ molar magnetic susceptibility) vs. $T$ was shown in Fig. 2 . The $\chi_{\mathrm{m}} T$ values were observed to increase slightly in response to the decrease of temperature and

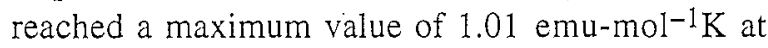
$45 \mathrm{~K}$. Below $45 \mathrm{~K}$, a sharp decline of $\chi_{\mathrm{m}} T$ values was detected. The decrease of $\chi_{\mathrm{m}} T$ values below 45 $\mathrm{K}$ may arise from intermolecular antiferromagnetic interactions between radicals in solid. Reciprocal magnetic susceptibilities of 2 in the temperature range of $2-10 \mathrm{~K}$ revealed a CurieWeiss behavior of spins with an antiferromagnetic coupling between spins of $\theta=-1.91 \mathrm{~K}$. The effective magnetic moment ( $\mu_{\text {eff }}$ ) value of $2.85 \mu_{\mathrm{B}}$ at $50-300 \mathrm{~K}$ is below a theoretical value of 3.00 $\mu_{\mathrm{B}}$ for $\mathrm{S}=3$ of a spin $1 / 2$ species.

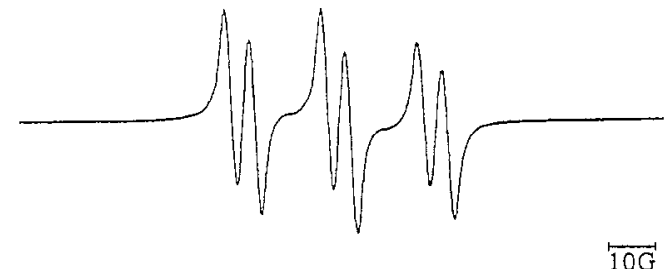

Fig. 1. ESR spectrum of the radical $\mathbf{1 b}$.

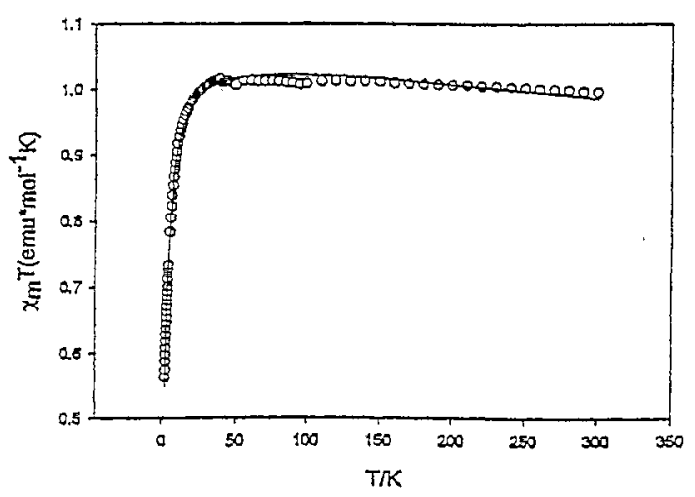

Fig. 2. The plot of $\chi_{\mathrm{m}} T$ vs. $T$ for the radical 2.

In conclusion, we have synthesized two stable nitroxyl triadicals ( 1 ) and ( 2 ) by either an addition reaction of alkyl Grignard reagents with the corresponding trinitrone molecules or the condensation reaction of 4-amino-2,2,6,6-tetramethylpiperidinyloxy with 1,3,5-benzenetricarboxaldehyde (trimesic aldehyde). The $\chi_{\mathrm{m}} T$ value was found to increase slightly as the temperature decreased. It reached a maximum value of 1.01 emu-mol-1 $^{-1} \mathrm{~K}$ at $45 \mathrm{~K}$ followed by a sharp decline of the $\chi_{\mathrm{m}} T$ value, indicating the existence of intermolecular antiferromagnetic interactions $(\theta=-1.91 \mathrm{~K})$ in this molecular system. The effective magnetic moment was found to be $2.85 \mu_{\mathrm{B}}$.

\section{References}

[1] D. Shiomi, M. Tamura, H. Sawa, R. Kato and M. Kinoshita, Synthetic Metals, 55-57 (1993) 3279.

[2] K. Awaga, T. Sugano and M. Kinoshita, $J$. Chem. Phys., (1986) 2211.

[3] L. Y. Chiang, L. Y. Wang and R. B. Upasani, Synthetic Metals., 71 (1995) 1801.

[4] K. Pan, C. R. Lin and T. I. Ho, Magan, Reson. Chem., 31 (1993) 632.

[5] J. S. Miller, A. J. Epstein and W. M. Reiff, Chem. Rev., 88 (1988) 201.

[6] E. G. Janzen and B. J. Blackburn, J. Am. Chem. Soc., 90 (1968) 5909.

[7] T. Ishida, H. Tomioka, H. Yoshikawa, M. Yasui, F.Iwasaki, H. Iwamura, N. Takeda and M. Ishikawa, Chem. Lett., (1994) 29. 